PROCEEDINGS OF THE

AMERICAN MATHEMATICAL SOCIETY

Volume 139, Number 9, September 2011, Pages 3271-3278

S 0002-9939(2011)10805-0

Article electronically published on April 6, 2011

\title{
SIMULTANEOUS METRIZABILITY OF COARSE SPACES
}

\author{
NICK WRIGHT \\ (Communicated by Alexander N. Dranishnikov)
}

\begin{abstract}
A metric space can be naturally endowed with both a topology and a coarse structure. We examine the converse to this. Given a topology and a coarse structure we give necessary and sufficient conditions for the existence of a metric giving rise to both of these. We conclude with an application to the construction of the coarse assembly map.
\end{abstract}

\section{INTRODUCTION}

A metric on a set $X$ naturally gives rise to both a topology and a coarse structure. These are to a large extent independent. Indeed, given a metric $d$ on $X$, one can define a metric $d_{t}$ by $d_{t}(x, y)=\min \{d(x, y), 1\}$, which produces the same topology as $d$ but contains no coarse geometric information. Alternatively, if one defines $d_{c}$ by $d_{c}(x, y)=\lceil d(x, y)\rceil$, where $\lceil s\rceil$ denotes the smallest integer greater than or equal to $s$, then it is straightforward to verify that this again defines a metric. In this case the metric produces the same coarse structure as $d$, and indeed it is quasi-isometric to $d$, while topologically this metric is discrete.

We will address the converse of this: under what conditions does there exist a metric on a set $X$ which simultaneously produces a given topology and coarse structure? Certainly the topology and coarse structure must each independently by metrizable, given say by metrics $d_{t}$ and $d_{c}$. Given a metric on $X$, the set $\{(x, y): d(x, y)<1\}$ is both open and controlled. Thus the topology and coarse structure cannot be completely independent: there must exist a controlled open neighbourhood of the diagonal. When this condition holds we say that the coarse structure is compatible with the topology. Remarkably, this is the only obstruction. The topology imposes no other constraints on the coarse structure and vice versa. Indeed we prove the following.

Theorem 2.5. Let $X$ be equipped with metrics $d_{t}, d_{c}$ with the coarse structure of $d_{c}$ compatible with the topology of $d_{t}$. Then there exists a metric $d$ on $X$ producing the same topology as $d_{t}$ and quasi-isometric to $d_{c}$.

Corollary 2.6. A set $X$ equipped with both a coarse structure and a topology is simultaneously metrizable if and only if the coarse structure is unital, weakly connected and countably generated, the topology is regular, Hausdorff and has a $\sigma$-locally finite base, and the coarse structure is compatible with the topology.

Received by the editors September 22, 2009 and, in revised form, August 13, 2010.

2010 Mathematics Subject Classification. Primary 46L85, 51F99.

Key words and phrases. Coarse geometry, metrizability, assembly map.

(C)2011 American Mathematical Society Reverts to public domain 28 years from publication 
As an application of this, we examine the construction of the coarse assembly map. The left hand side of the assembly map is the coarse $K$-homology $K X_{*}(X)$. This is constructed from $K$-homology using a sequence of simplicial complexes $N_{i}$ that in some sense approximate $X$. There is a subtlety here: the simplicial complexes, given their usual metric, need not be coarsely equivalent to $X$. We apply the metrizability theorem to rectify this problem, thus obtaining the coarse assembly map as the direct limit of maps

$$
K_{*}\left(N_{i}\right) \rightarrow K_{*}\left(C^{*}\left(N_{i}\right)\right) \cong K_{*}\left(C^{*}(X)\right) .
$$

\section{Coarse structures}

The notion of a coarse structure was introduced in [1]; see also [3], chapter 6 , and [7.

Definition 1.1. A coarse structure on a set $X$ is a collection $\mathfrak{E}$ of subsets of $X \times X$ satisfying the following axioms:

(i) If $A \in \mathfrak{E}$, then $A^{T}=\{(y, x):(x, y) \in A\}$ is in $\mathfrak{E}$.

(ii) If $A \in \mathfrak{E}$ and $B \in \mathfrak{E}$, then $A \circ B=\{(x, y): \exists z(x, z) \in A,(z, y) \in B\}$ is in $\mathfrak{E}$.

(iii) If $A \in \mathfrak{E}$ and $B \in \mathfrak{E}$, then $A \cup B$ is in $\mathfrak{E}$.

(iv) If $A \in \mathfrak{E}$ and $B \subset A$, then $B \in \mathfrak{E}$.

Elements of $\mathfrak{E}$ are referred to as controlled sets or entourages.

Definition 1.2. A coarse structure on a set $X$ is unital if the diagonal $\{(x, x)$ : $x \in X\}$ is an entourage, and it is weakly connected if all singletons are entourages.

Given a metric space $(X, d)$, there is a canonically associated coarse structure whose entourages are sets $E \subseteq X \times X$ with $d(x, y)$ bounded for $(x, y) \in E$.

Definition 1.3. Two metric spaces $(X, d),\left(X^{\prime}, d^{\prime}\right)$ are quasi-isometric if for some $A, B, R>0$ there is a map $f: X \rightarrow X^{\prime}$ such that

$$
\frac{1}{A} d(x, y)-B \leq d^{\prime}(f(x), f(y)) \leq A d(x, y)+B
$$

and every point of $X^{\prime}$ lies within distance $R$ of the image of $f$. The map $f$ is called a quasi-isometry.

Two metrics $d, d^{\prime}$ on a single space $X$ are said to be quasi-isometric if the identity map from $(X, d)$ to $\left(X, d^{\prime}\right)$ is a quasi-isometry.

We note that if $d$ and $d^{\prime}$ are quasi-isometric, then they induce the same coarse structure on $X$.

Definition 1.4. A coarse structure $\mathfrak{E}$ is said to be generated by $\mathfrak{S} \subset \mathfrak{E}$ if $\mathfrak{E}$ is the smallest coarse structure containing $\mathfrak{S}$. Equivalently, every element of $\mathfrak{E}$ is contained in a set produced by finitely many unions, compositions and transpositions of elements in $\mathfrak{S}$.

The following result was proven in [7, Theorem 2.55.

Theorem 1.5. A coarse structure on $X$ arises from a metric if and only if it is unital, weakly connected and countably generated. 
We will give a brief outline of the proof. The fact that metrics produce such coarse structures is completely elementary - the coarse structure is generated by the sets $A_{n}=\{(x, y): d(x, y)<n\}$. To show that such a coarse structure arises from a metric, take the given countable generating set $A_{1}, A_{2}, \ldots$, let $B_{0}$ be the diagonal, $B_{1}=A_{1} \cup A_{1}^{T} \cup B_{0}$, and recursively define $B_{n}=A_{n} \cup A_{n}^{T} \cup \bigcup_{i=1}^{n-1} B_{i} \circ B_{n-i}$ for $n>1$. It can then be shown that $d(x, y)=\min \left\{n:(x, y) \in B_{n}\right\}$ is a metric and that it produces the given coarse structure.

\section{Simultaneous metrizability}

As well as producing a coarse structure, a metric also gives rise to a topology. The question of whether a given topology arises from a metric is now well understood; see 4, section 5, for a full discussion of metrization theorems.

Definition 2.1. A collection of subsets of $X$ is $\sigma$-locally finite if it is a countable union of locally finite collections of subsets.

The following theorem was proved independently in [5, 8,

Theorem 2.2 (Nagata-Smirnov metrization theorem). A topological space $X$ is metrizable if and only if it is regular and Hausdorff, and the topology admits a $\sigma$-locally finite base.

We now make the following definition.

Definition 2.3. A set $X$ equipped with a topology and coarse structure is simultaneously metrizable if there exists a metric on $X$ giving rise to both the topology and the coarse structure.

If $X$ is a metric space, then the topology and coarse structure on $X$ satisfy a certain compatibility condition, viz. there exists an entourage which is an open neighbourhood of the diagonal. For instance the set of pairs $(x, y)$ with $d(x, y)<1$ is both an open neighbourhood of the diagonal and an entourage.

Note that the existence of an open entourage containing the diagonal implies that every entourage is contained in an open one: pre- and post-composing any entourage $A$ with an open entourage containing the diagonal will produce an open entourage containing $A$. Conversely, if every entourage is contained in an open one, and additionally the coarse structure is unital, then there is an open entourage containing the diagonal.

Definition 2.4. A coarse structure $\mathfrak{E}$ on a topological space $X$ is compatible with the topology if every entourage is contained in an open entourage.

Remark. This is one of the two axioms required for a coarse structure to be proper. The second axiom is that any subset $K$ of $X$ for which $K \times K$ is an entourage must be relatively compact. We however will only require compatibility: our theorem applies whether or not the coarse structure is proper.

For a set $X$ equipped with a topology and coarse structure, we now have certain obvious necessary conditions for $X$ to be simultaneously metrizable. Firstly both the metric and the topology must each independently be metrizable, and secondly the coarse structure must be compatible with the topology. We shall prove that these necessary conditions are in fact sufficient. 
Theorem 2.5. Let $X$ be equipped with metrics $d_{t}, d_{c}$ with the coarse structure of $d_{c}$ compatible with the topology of $d_{t}$. Then there exists a metric $d$ on $X$ producing the same topology as $d_{t}$ and quasi-isometric to $d_{c}$.

The theorem allows us to give necessary and sufficient conditions for simultaneous metrizability.

Corollary 2.6. A set $X$ equipped with both a coarse structure and a topology is simultaneously metrizable if and only if the coarse structure is unital, weakly connected and countably generated, the topology is regular, Hausdorff and has a $\sigma$ locally finite base, and the coarse structure is compatible with the topology.

Proof. The 'only if' direction is clear. For the converse, the hypotheses, along with Theorems 1.5 and 2.2. provide metrics $d_{t}$ and $d_{c}$ giving rise to the topology and coarse structure respectively. Now by Theorem 2.5 there is another metric $d$ giving rise to the same topology and which is quasi-isometric to $d_{c}$. Since it is quasi-isometric to $d_{c}$, it gives rise to the same coarse structure on $X$, hence $X$ is simultaneously metrizable.

Before proving the theorem we give a lemma which is useful for the construction of metrics.

Lemma 2.7. If $w(x, y)$ is a symmetric function on $X \times X$ with $w(x, x)=0$ and there exists a metric $d$ on $X$ with $d(x, y) \leq w(x, y)$ for all $x, y$, then there exists a maximal such metric. The maximal metric is given by the formula

$$
d^{\prime}(x, y)=\inf \left\{\sum_{i=1}^{n} w\left(x_{i-1}, x_{i}\right): n \in \mathbb{N}, x_{0}, \ldots, x_{n} \in X, x_{0}=x, x_{n}=y\right\} .
$$

Proof. For any metric $d$ with $d(x, y) \leq w(x, y)$ for all $x, y$ and for $n \in \mathbb{N}, x_{0}, \ldots, x_{n} \in$ $X, x_{0}=x, x_{n}=y$, we have $d(x, y) \leq \sum_{i=1}^{n} d\left(x_{i-1}, x_{i}\right)$ by the triangle inequality. On the other hand, $d\left(x_{i-1}, x_{i}\right) \leq w\left(x_{i-1}, x_{i}\right)$. Hence we deduce that $d(x, y) \leq$ $\sum_{i=1}^{n} w\left(x_{i-1}, x_{i}\right)$ for all such 'paths' $x_{0}, \ldots, x_{n}$ from $x$ to $y$. Thus for $d^{\prime}$ as above and any metric $d$ with $d(x, y) \leq w(x, y)$ we have $d(x, y) \leq d^{\prime}(x, y)$.

We must now show that $d^{\prime}$ is a metric. Certainly it is non-negative and symmetric. For three points $x, y, z$ in $X$, we have

$$
d^{\prime}(x, y)+d^{\prime}(y, z)=\inf \left\{\sum_{i=1}^{n} w\left(x_{i-1}, x_{i}\right): \begin{array}{l}
k, n \in \mathbb{N}, k<n, x_{i} \in X \\
x_{0}=x, x_{k}=y, x_{n}=z
\end{array}\right\} .
$$

This is greater than or equal to $d^{\prime}(x, z)$, thus we have the triangle inequality. Finally we note that as there exists at least one metric $d$ with $d(x, y) \leq w(x, y)$, we know that there is some metric $d$ with $d(x, y) \leq d^{\prime}(x, y)$. Thus whenever $x \neq y$ we have $d^{\prime}(x, y) \geq d(x, y)>0$.

We now proceed with the proof of the theorem.

Proof of Theorem 2.5. Without loss of generality we assume that the metric $d_{c}$ is uniformly discrete with $d_{c}(x, y) \geq 1$ for all $x \neq y$ (otherwise we can replace $d_{c}(x, y)$ by the quasi-isometric metric $\left.\left\lceil d_{c}(x, y)\right\rceil\right)$. By compatibility of the coarse structure with the topology there exists $U$ in $X \times X$ which is open with respect to $d_{t}$, contains the diagonal and is a $d_{c}$-entourage; i.e. for some $R$ we have $d_{c}(x, y)<R$ for all $(x, y) \in U$. 
Claim. There exists a metric $\delta$, topologically equivalent to $d_{t}$, and for which the 1-neighbourhood of the diagonal of $X \times X$ is an entourage. Specifically there exists $\delta$ with the property that $\delta(x, y)<1$ implies $d_{c}(x, y)<2 R$.

Proof of the Claim. As $U$ is open with respect to $d_{t}$, for each $x \in X$ there exists $\varepsilon>0$ such that if $d_{t}(x, y)<\varepsilon$, then $(x, y) \in U$. Let $\varepsilon_{x}$ be the supremum of the set of such $\varepsilon$, if this is less than 1 , and let $\varepsilon_{x}=1$ otherwise. By construction, if $d_{t}(x, y)<\varepsilon_{x}$, then $(x, y) \in U$; hence $d_{c}(x, y)<R$. We will define $\delta$ to be the maximal metric such that $\delta(x, y) \leq \frac{d_{t}(x, y)}{\max \left\{\varepsilon_{x}, \varepsilon_{y}\right\}}$ for all $x, y$. By Lemma 2.7 such a metric exists provided that there is at least one metric satisfying this inequality, and we note that the inequality is satisfied by $d_{t}$ since $\max \left\{\varepsilon_{x}, \varepsilon_{y}\right\} \leq 1$. As $\delta$ is the maximal such metric, we note that for each $x$ we have $d_{t}(x, y) \leq \delta(x, y) \leq$ $d_{t}(x, y) / \varepsilon_{x}$ which shows that $\delta$ gives rise to the same topology as $d_{t}$.

Now suppose that $\delta(x, y)<1$. Then there must be a sequence $x=x_{0}, x_{1}, \ldots, x_{n}$ $=y$ such that $\sum_{i=1}^{n} \frac{d_{t}\left(x_{i-1}, x_{i}\right)}{\varepsilon_{i}}<1$ where $\varepsilon_{i}=\max \left\{\varepsilon_{x_{i-1}}, \varepsilon_{x_{i}}\right\}$, as $\delta(x, y)$ is given by the infimum of these sums. Let $i_{0}$ be the value of $i$ for which $\varepsilon_{i}$ is greatest. Then

$$
d_{t}\left(x, x_{i_{0}}\right) \leq \sum_{i=1}^{i_{0}} d_{t}\left(x_{i-1}, x_{i}\right) \leq \varepsilon_{i_{0}} \sum_{i=1}^{i_{0}} \frac{d_{t}\left(x_{i-1}, x_{i}\right)}{\varepsilon_{i}}<\varepsilon_{i_{0}} .
$$

Similarly $d_{t}\left(x_{i_{0}}, y\right)<\varepsilon_{i_{0}}$. Hence it follows that $d_{c}\left(x, x_{i_{0}}\right)<R, d_{c}\left(x_{i_{0}}, y\right)<R$, and so if $\delta(x, y)<1$, then $d_{c}(x, y)<2 R$. This proves the claim.

We now define a metric $d$ which is topologically equivalent to $d_{t}$ and quasiisometric to $d_{c}$. We define $d$ to be the maximal metric such that

(i) $d(x, y) \leq d_{c}(x, y)$,

(ii) $d(x, y) \leq \delta(x, y)$ when $\delta(x, y) \leq \frac{1}{2}$.

As before the existence of this metric is guaranteed by Lemma 2.7 provided that there exists one such metric, and we note that the metric $\min \{\delta(x, y), 1\}$ has this property, since $d_{c}(x, y) \geq 1$ whenever $x \neq y$. On small scales the metric $d$ agrees with $\delta$ : indeed $d(x, y)=\delta(x, y)$ whenever one of these is at most $\frac{1}{2}$, which again follows from the observation that $d_{c}(x, y) \geq 1$ whenever $x \neq y$. We deduce that $d$ gives rise to the same topology as $\delta$ and hence $d_{t}$.

Now suppose that $x, y \in X$ with $d(x, y)<k \in \mathbb{N}$. Then there exists a sequence $x=x_{0}, x_{1}, \ldots, x_{n}=y$ such that $\sum_{i=1}^{n} c_{i}<k$, where for each $i$, either (i) $c_{i}=$ $d_{c}\left(x_{i-1}, x_{i}\right)$ or (ii) $c_{i}=\delta\left(x_{i-1}, x_{i}\right)$ with $c_{i}<\frac{1}{2}$. Let $I$ be the set of $i$ for which (i) holds, and partition $\{1,2, \ldots, n\} \backslash I$ into maximal subsets of consecutive integers. For $i \in I$ we have $c_{i} \geq 1$, so $I$ has cardinality at most $k-1$, and hence there are at most $k$ sets in the partition. Let $J=\{p+1, \ldots, q\}$ be one such subset, and let $c_{J}=\sum_{i=p+1}^{q} c_{i}$. We will now make shortcuts in the 'path' $x_{p}, x_{p+1}, \ldots, x_{q}$. Each step $d\left(x_{i-1}, x_{i}\right)$ is at most $\frac{1}{2}$; we will pick out a subsequence so that each step (except possibly the last) has length between $\frac{1}{2}$ and 1 . Formally, we pick $j_{0}=p, \ldots, j_{m}=q$ as an increasing sequence, such that for $l=1, \ldots, m$ we have $\delta\left(x_{j_{l-1}}, x_{j_{l}}\right)<1$ and for $l<m$ we have $\frac{1}{2} \leq \delta\left(x_{j_{l-1}}, x_{j_{l}}\right)$. Note that the final step may be less than $\frac{1}{2}$. The sum $\sum_{l=1}^{m-1} \delta\left(x_{j_{l-1}}, x_{j_{l}}\right)$ is at most $c_{J}$ and hence $c_{J} \geq \frac{1}{2}(m-1)$, since each summand is at least $\frac{1}{2}$. Rearranging we have $m \leq 2 c_{J}+1$. On the other hand, for each $l$ we have $\delta\left(x_{j_{l-1}}, x_{j_{l}}\right)<1$, so $d_{c}\left(x_{j_{l-1}}, x_{j_{l}}\right)<2 R$. Thus $d_{c}\left(x_{p}, x_{q}\right)<2 R m \leq 2 R\left(2 c_{J}+1\right)$. 
Now we reassemble the path. For each $i$ in $I$ we have $c_{i}=d_{c}\left(x_{i-1}, x_{i}\right)$, so $\sum_{i \in I} d_{c}\left(x_{i-1}, x_{i}\right)<k$. On the other hand for each set $J=\{p+1, \ldots, q\}$ in the partition of $\{1,2, \ldots, n\} \backslash I$ we have $d_{c}\left(x_{p}, x_{q}\right)<2 R\left(2 c_{J}+1\right)$. There are at most $k$ of these, and the sum of the terms $c_{J}$ is at most $k$. Hence the sum of $d_{c}\left(x_{p}, x_{q}\right)$ over all sets in the partition is at most $6 R k$. We thus conclude that if $d(x, y)<k$, then $d_{c}(x, y)<(6 R+1) k$. This is true for all integers $k$; thus we have $d(x, y) \leq d_{c}(x, y)<(6 R+1)(d(x, y)+1)$. These inequalities show that $d$ is quasi-isometric to $d_{c}$, which completes the proof.

\section{THE ASSEMBLY MAP}

We now discuss applications of simultaneous metrizability in the context of the coarse assembly map, introduced in [2, 10.

Let $X$ be a set equipped with a coarse structure. Then the assembly map for $X$ is a homomorphism

$$
K X_{*}(X) \rightarrow K_{*}\left(C^{*}(X)\right)
$$

where $K X_{*}(X)$ is the coarse $K$-homology of $X$ and $C^{*}(X)$ is the Roe algebra of $X$. Coarse $K$-homology, the Roe algebra and the assembly map are defined in [6]; see also [3, 9].

Let $\left(X, d_{X}\right)$ be a metric space. Then there is a homomorphism $K_{*}(X) \rightarrow$ $K_{*}\left(C^{*}(X)\right)$ defined by the boundary map in a certain $K$-theory exact sequence (cf. [6, 3, 9]). This map is not an isomorphism in general since the left hand side is a topological invariant, while the right hand side is a coarse invariant. To define the assembly map one 'coarsens' the left hand side.

Let $\mathcal{U}_{i}$ be a sequence of covers of $X$, let $D_{i}$ denote the supremum of the diameters of $U$ for $U \in \mathcal{U}_{i}$, and let $\lambda_{i}$ denote the Lebesgue number 11 of the cover $\mathcal{U}_{i}$. Suppose that $D_{i} \rightarrow \infty$, and for each $i$ we have $D_{i} \leq \lambda_{i+1}$. This ensures that $\mathcal{U}_{i+1}$ coarsens $\mathcal{U}_{i}$ in the sense that for each $U$ in $\mathcal{U}_{i}$ there exists $U^{\prime}$ in $\mathcal{U}_{i+1}$ such that $U \subseteq U^{\prime}$. A sequence $\mathcal{U}_{i}$ with these properties is called an anti-ČCh sequence for $X$. It is straightforward to show that these exist; indeed, taking $\mathcal{U}_{i}$ to be the cover of $X$ by all balls of radius $2^{i}$, one has $D_{i} \leq 2^{i+1} \leq \lambda_{i+1}$.

For each $i$ define $N_{i}$ to be the nerve of the cover $\mathcal{U}_{i}$ : this is the simplicial complex with a vertex $[U]$ for each element $U$ of $\mathcal{U}_{i}$, and with $\left[U_{0}, U_{1}, \ldots, U_{n}\right]$ defining an $n$-simplex when $U_{0} \cap \cdots \cap U_{n}$ is non-empty. The simplicial complex $N_{i}$ is given the path metric which restricts on each simplex to the standard spherical metric. For each $U$ in $\mathcal{U}_{i}$ choose an element $U^{\prime}$ in $\mathcal{U}_{i+1}$ such that $U \subseteq U^{\prime}$. The map taking the vertex $[U]$ in $N_{i}$ to the vertex [ $\left.U^{\prime}\right]$ in $N_{i+1}$ is simplicial, since $U_{0} \cap \cdots \cap U_{n}$ is non-empty and $U_{i}^{\prime} \supseteq U_{i}$ implies that $U_{0}^{\prime} \cap \cdots \cap U_{n}^{\prime}$ is non-empty.

Now for each $i$ we have a homomorphism $K_{*}\left(N_{i}\right) \rightarrow K_{*}\left(C^{*}\left(N_{i}\right)\right)$ (the boundary map as above). Using the simplicial maps $N_{i} \rightarrow N_{i+1}$ one can take the direct limits. By definition the coarse $K$-homology, $K X_{*}(X)$, is the direct limit $\underset{\lim }{\longrightarrow} K_{*}\left(N_{i}\right)$. Hence there is an assembly map

$$
K X_{*}(X) \rightarrow \underline{\lim } K_{*}\left(C^{*}\left(N_{i}\right)\right) .
$$

As the author observed in 9], the identification of the direct limit $\lim _{\rightarrow} K_{*}\left(C^{*}\left(N_{i}\right)\right)$ with $K_{*}(X)$ is non-trivial. In particular, for $N_{i}$ equipped with the spherical path

\footnotetext{
${ }^{1}$ The Lebesgue number of a cover $\mathcal{U}$ is the supremum of $d \in \mathbb{R}$ such that every set of diameter at most $d$ is a subset of some $U \in \mathcal{U}$.
} 
metric, it is not true in general that $K_{*}\left(C^{*}\left(N_{i}\right)\right)$ is isomorphic to $K_{*}(X)$ for each $i$.

Making use of simultaneous metrizability it is possible to remedy this problem. First we construct a metric on the nerves which is compatible with the spherical metric, but which has the correct coarse geometry.

Proposition 3.1. Let $\mathcal{U}$ be a cover of $X$ of diameter at most $D$, let $N$ be the nerve of $\mathcal{U}$, and let $d_{s}$ denote the spherical path metric on $N$. For each $x \in X$ choose an element $U_{x}$ of $\mathcal{U}$ such that $x \in U_{x}$. Then there is another metric $d_{c}$ on $N$ such that the map $F:\left(X, d_{X}\right) \rightarrow\left(N, d_{c}\right)$ defined by $x \mapsto\left[U_{x}\right]$ is a coarse equivalence and the $d_{c}$ coarse structure is compatible with the $d_{s}$ topology on $N$.

Proof. Define $G: N \rightarrow X$ as follows. Given $y \in N$, let $\left[U_{0}, \ldots, U_{n}\right]$ be the minimal simplex containing $y$. We note that $U_{0} \cap \cdots \cap U_{n}$ is non-empty by definition. Pick a point in $U_{0} \cap \cdots \cap U_{n}$, and define $G(y)$ to be this point. Then the map $G$ has the property that $d_{X}(G(F(x)), x) \leq D$, since $G(F(x))$ must lie in $U_{x}$ for each $x$. Hence $G \circ F$ is close to the identity on $X$.

Let $d_{\text {disc }}$ denote the discrete metric on $N$, and define $d_{c}\left(y, y^{\prime}\right)=d_{X}\left(G(y), G\left(y^{\prime}\right)\right)+$ $d_{\text {disc }}\left(y, y^{\prime}\right)$. We observe that $d_{c}$ is a metric. First note that the triangle inequality for the function $d_{X}\left(G(y), G\left(y^{\prime}\right)\right)$ on $N \times N$ follows immediately from the triangle inequality for the metric $d_{X}$, and $d_{c}$ is obtained by adding the discrete metric to this. Hence $d_{c}$ satisfies the triangle inequality. Positivity and symmetry of $d_{c}$ are immediate.

We will now show that the maps $F, G$ give a coarse equivalence between $X$ and $N$. For $y, y^{\prime}$ in $N$ we have $d_{X}\left(G(y), G\left(y^{\prime}\right)\right) \leq d_{c}\left(y, y^{\prime}\right)$ by definition, while for $x, x^{\prime}$ in $X$ we have $d_{c}\left(F(x), F\left(x^{\prime}\right)\right) \leq d_{X}\left(G(F(x)), G\left(F\left(x^{\prime}\right)\right)\right)+1$. We have already noted that $d_{X}(G(F(x)), x) \leq D$ for each $x$, hence

$$
d_{c}\left(F(x), F\left(x^{\prime}\right)\right) \leq d\left(x, x^{\prime}\right)+2 D+1 .
$$

The maps $F, G$ are thus bornologous. We have observed that $G \circ F$ is close to the identity on $X$, thus it remains to check that $F \circ G$ is close to the identity on $N$, as coarse properness for $F$ will then follow from the fact that $G$ is bornologous and vice versa. We have

$$
d_{c}(F(G(y)), y) \leq d_{X}(G(F(G(y)), G(y))+1 \leq D+1
$$

Hence $F \circ G$ is close to the identity and $X, N$ are coarsely equivalent as claimed.

To complete the proof, we must show that the $d_{c}$ coarse structure is compatible with the $d_{s}$ topology. In other words we must find a subset $E$ of $N \times N$ which is an open neighbourhood of the diagonal for the $d_{s}$ topology and on which $d_{c}$ is bounded. Define

$$
E=\bigcup\{S \times S: S \text { an open star in } N\}
$$

Certainly this is an open neighbourhood of the diagonal. Now for any $\left(y, y^{\prime}\right) \in E$ we have $y, y^{\prime} \in S$ for $S$ some open star of $N$. As $y, y^{\prime}$ lie in the same star, the minimal simplices containing $y, y^{\prime}$ must have some common vertex $[U]$. By definition of the map $G$ we have $G(y), G\left(y^{\prime}\right)$ in $U$; hence

$$
d_{c}\left(y, y^{\prime}\right) \leq d_{X}\left(G(y), G\left(y^{\prime}\right)\right)+1 \leq D+1 .
$$

Thus $d_{c}$ is bounded on $E$, which completes the proof.

Using Proposition 3.1 to construct a metric $d_{c}^{i}$ on $N_{i}$ which is compatible with the spherical metric $d_{s}^{i}$, and applying Theorem 2.5 we obtain the following. 
Corollary 3.2. Let $\mathcal{U}_{i}$ be an anti- $\check{C}$ ech sequence. Then each space $N_{i}$ can be equipped with a metric which gives rise to the usual topology on $N_{i}$ and such that $N_{i}$ is coarsely equivalent to $X$. Using these metrics we have homomorphisms

$$
K_{*}\left(N_{i}\right) \rightarrow K_{*}\left(C^{*}\left(N_{i}\right)\right) \cong K_{*}\left(C^{*}(X)\right) .
$$

The direct limit of these defines the coarse assembly map

$$
K X_{*}(X) \rightarrow K_{*}\left(C^{*}(X)\right) .
$$

\section{REFERENCES}

[1] N. Higson, E.K. Pedersen, and J. Roe, $C^{*}$-algebras and controlled topology. K-theory, 11 (1997), no. 3, 209-239. MR1451755 (98g:19009)

[2] N. Higson and J. Roe, On the coarse Baum-Connes conjecture. In Novikov Conjectures, Index Theorems, and Rigidity, Volume 2 (S.C. Ferry, A. Ranicki, and J. Rosenberg, editors), vol. 227 of LMS Lecture Note Series, 285-300. Cambridge University Press (1995). MR.1388312 (97f:58127)

[3] N. Higson and J. Roe, Analytic K-homology. Oxford Mathematical Monographs, Oxford University Press (2000). MR1817560 (2002c:58036)

[4] K. Nagami, Dimension Theory. Pure and Applied Mathematics, vol. 37, Academic Press (1970). MR0271918 (42:6799)

[5] J. Nagata, On a necessary and sufficient condition of metrizability. J. Inst. Polytech. Osaka City Univ. Ser. A. Math., 1 (1950), 93-100. MR0043448 (13:264e)

[6] J. Roe, Index theory, coarse geometry and the topology of manifolds. Regional Conference Series on Mathematics, vol. 90, CBMS Conference Proceedings, American Mathematical Society (1996). MR.1399087 (97h:58155)

[7] J. Roe, Lectures on Coarse Geometry. University Lecture Series, vol. 31, American Mathematical Society (2003). MR.2007488 (2004g:53050)

[8] Y. Smirnov, A necessary and sufficient condition for metrizability of a topological space. Doklady Akad. Nauk SSSR (N.S.), 77 (1951), 197-200. MR0041420(12:845c)

[9] N.J. Wright, The coarse Baum-Connes conjecture via $C_{0}$ coarse geometry. J. Funct. Anal., 220 (2005), no. 2, 265-303. MR2119281 (2006d:58023)

[10] G. Yu. On the coarse Baum-Connes conjecture. K-theory, 9 (1995), 199-221. MR.1344138 (96k:58214)

Mathematics, University of Southampton, University Road, Southampton, SO17 1BJ, UNITED KINGDOM

E-mail address: wright@soton.ac.uk 